\title{
Quick NIR Based Method for Ascertaining Coffee and Chicory Percentage in a Mixture
}

\author{
Nanishankar V.Harohally*, Cyril Thomas
}




\section{Table of contents}

\begin{tabular}{|c|c|}
\hline Details & Page no \\
\hline $\begin{array}{l}\text { Table 1: Effect of different preprocessing techniques on calibration and validation of } \\
\text { method for Robusta coffee and chicory mixture }\end{array}$ & 3 \\
\hline $\begin{array}{l}\text { Table 2: Effect of different preprocessing techniques on calibration and validation of } \\
\text { method for Robusta and Arabica }(1: 1) \text { coffee and chicory mixture }\end{array}$ & 4 \\
\hline $\begin{array}{l}\text { Table 3: Effect of different preprocessing techniques on calibration and validation of } \\
\text { method for Instant coffee \& chicory mixture }\end{array}$ & 5 \\
\hline Table 4: Representative functional groups in the frequency range $4100-9100 \mathrm{~cm}^{-1}$ & 6 \\
\hline NIR spectra of instant coffee and chicory mixture with no processing & 7 \\
\hline $\begin{array}{l}\text { NIR spectra of instant coffee and chicory mixture with first derivative and vector } \\
\text { normalization processing }\end{array}$ & 8 \\
\hline $\begin{array}{l}\text { Cross validation curves for instant coffee and chicory mixture with first derivative and } \\
\text { vector normalization processing }\end{array}$ & $9-10$ \\
\hline $\begin{array}{l}\text { NIR spectra of instant coffee and chicory mixture with multiplicative scattering } \\
\text { correction processing }\end{array}$ & 11 \\
\hline $\begin{array}{l}\text { Cross validation curves for instant coffee and chicory mixture with multiplicative } \\
\text { scattering correction processing }\end{array}$ & $12-13$ \\
\hline NIR spectra of (Arabica plus Robusta) coffee and chicory mixture with no processing & 14 \\
\hline $\begin{array}{l}\text { NIR spectra of (Arabica plus Robusta) coffee and chicory mixture with second } \\
\text { Derivative processing }\end{array}$ & 15 \\
\hline $\begin{array}{l}\text { Cross validation curves for (Arabica plus Robusta) coffee and chicory mixture } \\
\text { with second derivative processing }\end{array}$ & $16-17$ \\
\hline NIR spectra of Robusta coffee and chicory mixture with no processing & 18 \\
\hline NIR spectra of Robusta coffee and chicory mixture with second derivative processing & 19 \\
\hline $\begin{array}{l}\text { Cross validation curves for Robusta coffee and chicory mixture with second } \\
\text { derivative processing }\end{array}$ & $20-21$ \\
\hline NIR spectra of Arabica coffee and chicory mixture with no processing & 22 \\
\hline NIR spectra of Arabica coffee and chicory mixture with second derivative processing & 23 \\
\hline $\begin{array}{l}\text { Cross validation curves for Arabica coffee and chicory mixture with second derivative } \\
\text { processing }\end{array}$ & $24-25$ \\
\hline
\end{tabular}


Table 1: Effect of different preprocessing techniques on calibration and validation of method for Robusta coffee and chicory mixture

\begin{tabular}{|c|c|c|c|c|c|}
\hline $\begin{array}{l}\text { Preprocessing } \\
\text { technique }\end{array}$ & $\begin{array}{l}\mathbf{R}^{2} \\
\text { calibration }\end{array}$ & RMSEE & $\begin{array}{l}\mathbf{R}^{2} \\
\text { validation }\end{array}$ & $\begin{array}{l}\text { RMS } \\
\text { CV }\end{array}$ & PLS factor \\
\hline No preprocessing & 99.96 & 0.73 & 99.89 & 1.03 & 8 \\
\hline First derivative & 99.87 & 0.59 & 99.92 & 0.88 & 6 \\
\hline Second derivative & 99.97 & 0.54 & 99.82 & 1.29 & 5 \\
\hline $\begin{array}{l}\text { First derivative }+ \text { Straight } \\
\text { line substation }\end{array}$ & 99.99 & 0.28 & 99.89 & 1.03 & 8 \\
\hline $\begin{array}{l}\text { First derivative }+ \text { Vector } \\
\text { normalization }\end{array}$ & 99.98 & 0.51 & 99.93 & 0.84 & 6 \\
\hline First derivative $+\mathrm{MSC}$ & 99.98 & 0.44 & 99.93 & 0.82 & 6 \\
\hline Vector normalization & 99.98 & 0.54 & 99.91 & 0.95 & 8 \\
\hline $\begin{array}{l}\text { Multiplicative scattering } \\
\text { correction }\end{array}$ & 99.97 & 0.61 & 99.91 & 0.98 & 8 \\
\hline Straight line substation & 99.96 & 0.74 & 99.9 & 0.99 & 8 \\
\hline Min-Max normalization & 99.98 & 0.55 & 99.91 & 0.96 & 9 \\
\hline
\end{tabular}


Table 2: Effect of different preprocessing techniques on calibration and validation of method for Robusta and Arabica (1:1) coffee and chicory mixture

\begin{tabular}{|l|l|l|l|l|l|}
\hline $\begin{array}{l}\text { Preprocessing } \\
\text { technique }\end{array}$ & $\begin{array}{l}\mathbf{R}^{\mathbf{2}} \\
\text { calibration }\end{array}$ & $\mathbf{R M S E E}$ & $\begin{array}{l}\mathbf{R}^{2} \\
\text { validation }\end{array}$ & $\mathbf{R M S C V}$ & PLS factor \\
\hline No preprocessing & 99.81 & 1.51 & 99.66 & 1.79 & 7 \\
\hline First derivative & 99.99 & 0.35 & 99.85 & 1.13 & 8 \\
\hline Second derivative & 99.93 & 0.82 & 99.68 & 1.62 & 4 \\
\hline $\begin{array}{l}\text { First derivative + Straight } \\
\text { line subtraction }\end{array}$ & 99.99 & 0.28 & 99.87 & 1.06 & 8 \\
\hline $\begin{array}{l}\text { First derivative +Vector } \\
\text { normalization }\end{array}$ & 99.99 & 0.29 & 99.93 & 0.82 & 8 \\
\hline First derivative +MSC & 99.99 & 0.43 & 99.83 & 1.31 & 7 \\
\hline Vector normalization & 99.97 & 0.58 & 99.94 & 0.78 & 7 \\
\hline $\begin{array}{l}\text { Multiplicative scattering } \\
\text { correction }\end{array}$ & 99.95 & 0.73 & 99.9 & 0.94 & 7 \\
\hline Straight line subtraction & 99.99 & 0.41 & 99.87 & 1.19 & 9 \\
\hline Min-Max normalization & 99.98 & 0.48 & 99.86 & 1.09 & 9 \\
\hline
\end{tabular}


Table 3: Effect of different preprocessing techniques on calibration and validation of method for Instant coffee \& chicory mixture

\begin{tabular}{|l|l|l|l|l|l|}
\hline $\begin{array}{l}\text { Preprocessing } \\
\text { technique }\end{array}$ & $\begin{array}{l}\mathbf{R}^{\mathbf{2}} \\
\text { calibration }\end{array}$ & RMSEE & $\begin{array}{l}\mathbf{R}^{\mathbf{2}} \\
\text { validation }\end{array}$ & $\begin{array}{l}\text { RMS } \\
\text { CV }\end{array}$ & $\begin{array}{l}\text { PLS } \\
\text { factor }\end{array}$ \\
\hline No preprocessing & 99.95 & 0.74 & 99.92 & 0.88 & 8 \\
\hline First derivative & 99.96 & 0.68 & 99.9 & 0.95 & 6 \\
\hline Second derivative & 99.95 & 0.70 & 99.75 & 1.5 & 5 \\
\hline $\begin{array}{l}\text { First derivative + Straight } \\
\text { line substation }\end{array}$ & 99.96 & 0.62 & 99.92 & 0.84 & 6 \\
\hline $\begin{array}{l}\text { First derivative +Vector } \\
\text { normalization }\end{array}$ & 99.9 & 0.99 & 99.88 & 1.06 & 3 \\
\hline First derivative +MSC & 99.9 & 0.99 & 99.88 & 1.08 & 3 \\
\hline Vector normalization & 99.99 & 0.40 & 99.95 & 0.65 & 9 \\
\hline $\begin{array}{l}\text { Multiplicative scattering } \\
\text { correction }\end{array}$ & 99.99 & 0.33 & 99.96 & 0.64 & 9 \\
\hline Straight line substation & 99.95 & 0.76 & 99.91 & 0.94 & 8 \\
\hline Min-Max normalization & 99.98 & 0.50 & 99.96 & 0.67 & 8 \\
\hline
\end{tabular}


Table 4: Representative functional groups in the frequency range $4100-9100 \mathrm{~cm}^{-1}$

\begin{tabular}{|c|c|c|c|}
\hline Groups & $\begin{array}{l}\text { Nature of } \\
\text { bands }\end{array}$ & Frequency $\left(\mathrm{cm}^{-1}\right)$ & Assignment \\
\hline \multirow{6}{*}{$\begin{array}{l}\mathrm{CH}_{3} \\
\text { (methyl) }\end{array}$} & \multirow[t]{2}{*}{ Combination } & $4380-4400$ & $\mathrm{CH}$ stretching $+\mathrm{CH}$ bending \\
\hline & & $7330-7380$ & 2x CH stretching+ $\mathrm{CH}$ bending \\
\hline & \multirow[t]{2}{*}{$1^{\text {st }}$ overtone } & $5850-5780$ & $1^{\text {st }}$ overtone of anti-symmetric stretching \\
\hline & & $5600-5650$ & $1^{\text {st }}$ overtone of symmetric stretching \\
\hline & \multirow[t]{2}{*}{$2^{\text {nd }}$ overtone } & $8580-8700$ & $2^{\text {nd }}$ overtone of anti-symmetric stretching \\
\hline & & $8330-8400$ & $2^{\text {nd }}$ overtone of symmetric stretching \\
\hline \multirow{6}{*}{$\begin{array}{l}\mathrm{CH}_{2} \\
\text { (Methylene) }\end{array}$} & Combination & $4290-4310$ & $\mathrm{CH}$ stretching $+\mathrm{CH}$ bending \\
\hline & & $7040-7090$ & 2X CH stretching+ 3x CH bending \\
\hline & $1^{\text {st }}$ overtone & $5710-5760$ & $1^{\text {st }}$ overtone of anti-symmetric stretching \\
\hline & & $5570-5620$ & $1^{\text {st }}$ overtone of symmetric stretching \\
\hline & $2^{\text {nd }}$ overtone & $8470-8550$ & $2^{\text {nd }}$ overtone of anti-symmetric stretching \\
\hline & & $8260-8330$ & $2^{\text {nd }}$ overtone of symmetric stretching \\
\hline \multirow{5}{*}{$\begin{array}{l}\mathrm{CH} \\
\text { (aromatic) }\end{array}$} & \multirow[t]{3}{*}{ Combination } & $6900-6940$ & 2x CH stretching $+\mathrm{CH}$ bending \\
\hline & & $7040-7090$ & 2x CH stretching+ 3x CH bending \\
\hline & & $9220-9350$ & 2x CH stretching+ 3x CH bending \\
\hline & $1^{\text {st }}$ overtone & $5920-5950$ & None \\
\hline & $2^{\text {nd }}$ overtone & $8770-8850$ & None \\
\hline $\begin{array}{l}\mathrm{C}=\mathrm{O} \\
\text { (Ketone) }\end{array}$ & $2^{\text {nd }}$ overtone & $5110-1230$ & None \\
\hline $\begin{array}{l}\text { CHO } \\
\text { aldehyde }\end{array}$ & Combination & $4520-4570$ & $\mathrm{CH}$ stretching $+\mathrm{C}=\mathrm{O}$ streching \\
\hline \multirow{3}{*}{$\begin{array}{l}\text { Free } \\
\text { OH(alcohol) }\end{array}$} & Combination & $4780-4850$ & $\mathrm{OH}$ stretching $+\mathrm{OH}$ bending \\
\hline & $1^{\text {st }}$ overtone & $7020-7170$ & \\
\hline & $2^{\text {nd }}$ overtone & $4180-4220$ & $2^{\text {nd }}$ overtone of $\mathrm{OH}$ bending \\
\hline
\end{tabular}


NIR spectra of instant coffee and chicory mixture with no processing

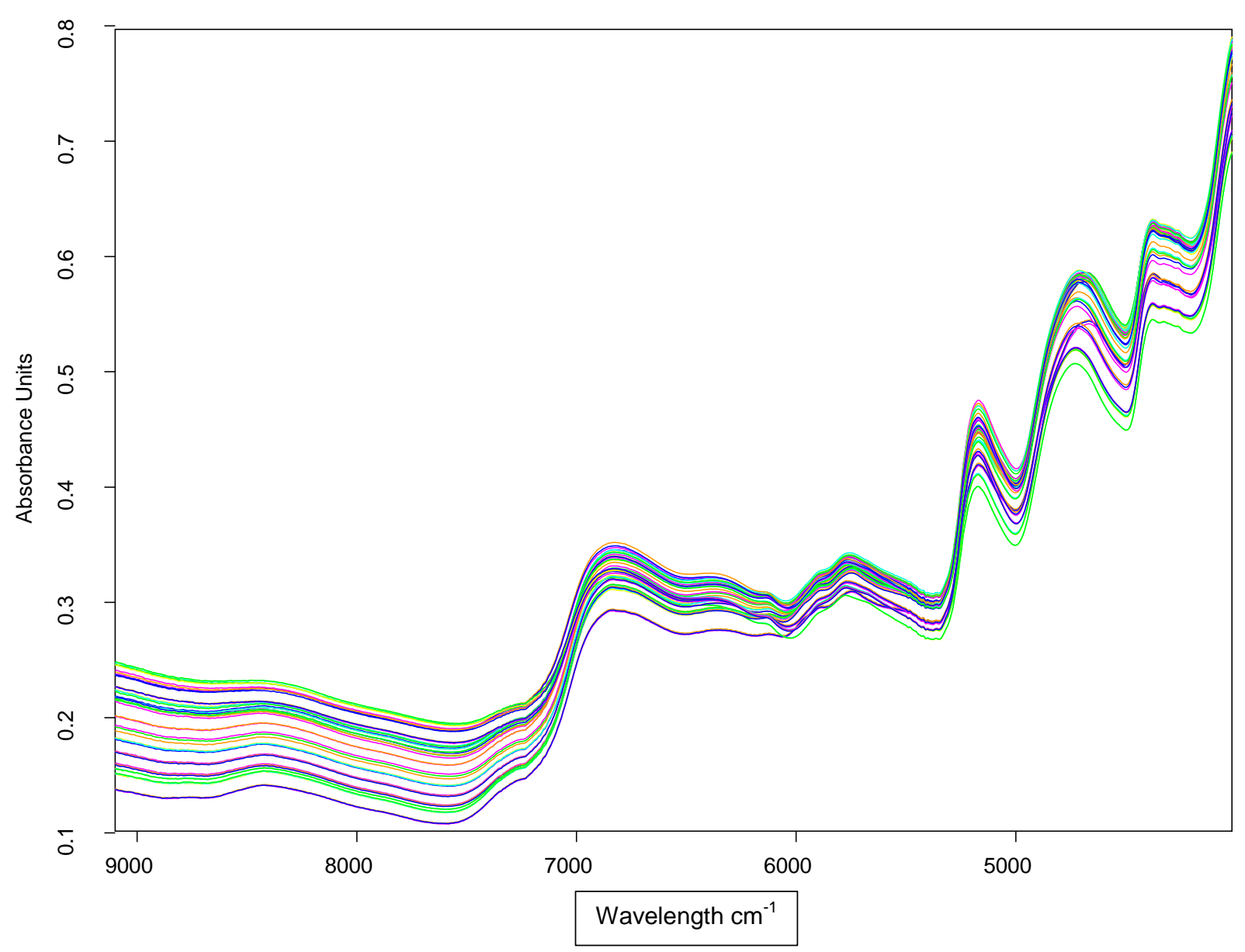


NIR spectra of instant coffee and chicory mixture with first derivative and multiplicative scattering correction processing

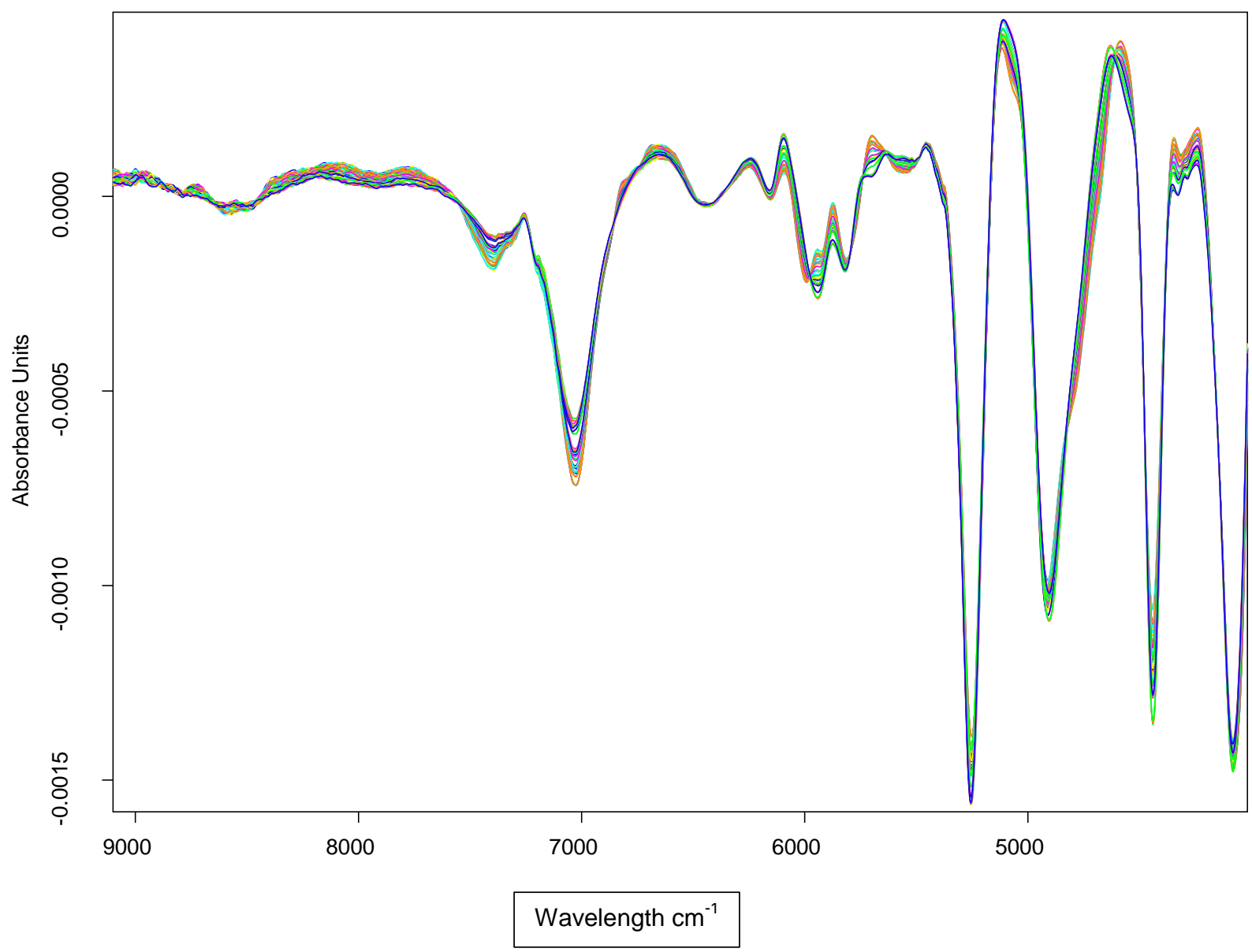


Prediction vs True / Coffee [\%] / Cross Validation

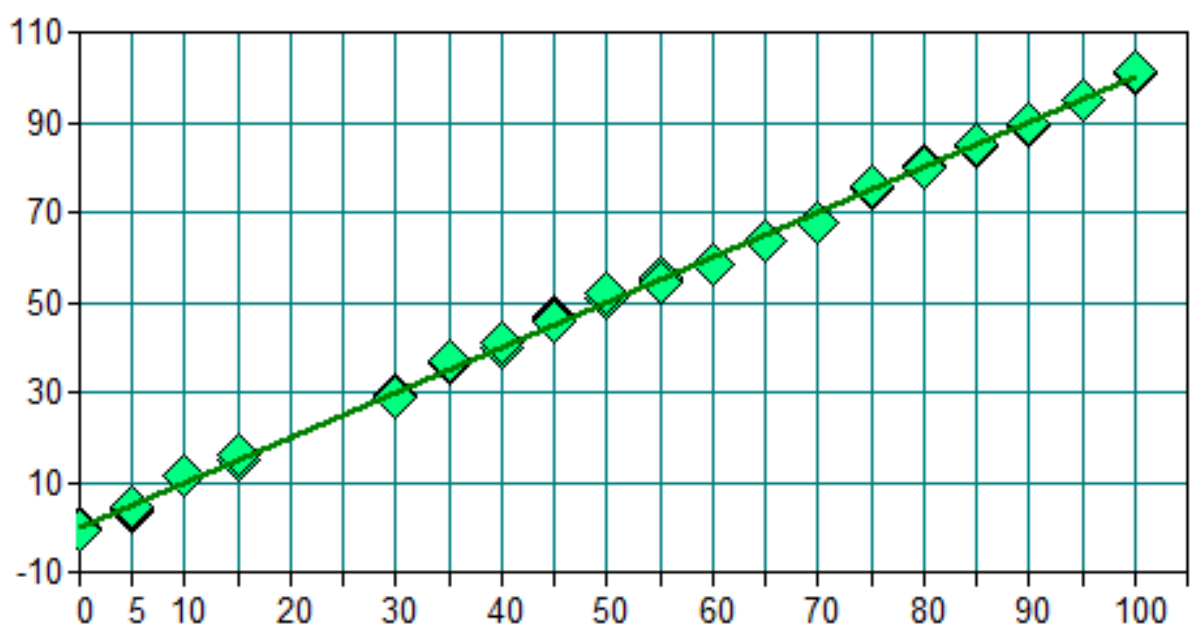

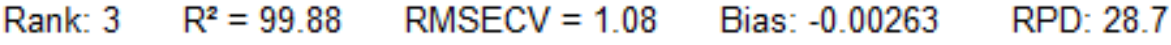
Validation No $5+$ IncoffchnofdervMSC.q2

Difference vs True / Coffee [\%] / Cross Validation

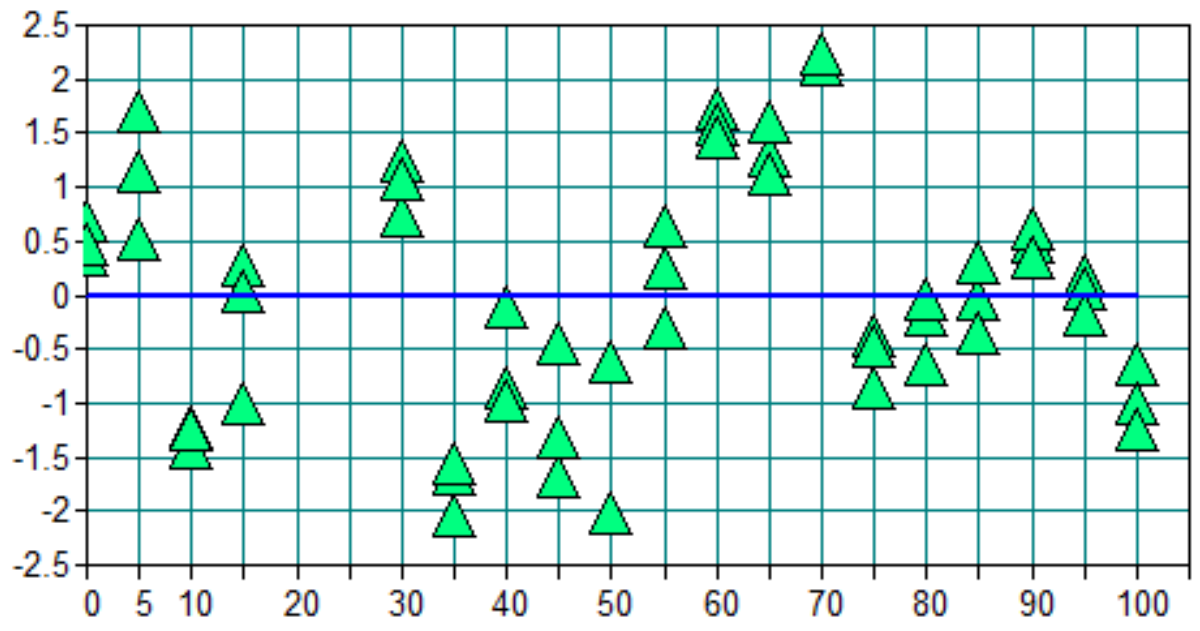

$\begin{array}{lllll}\text { Rank: } 3 & \mathrm{R}^{2}=99.88 & \text { RMSECV }=1.08 & \text { Bias: }-0.00263 & \text { RPD: } 28.7\end{array}$

Validation No $5+$ IncoffchnofdervMSC.q2 
Mah. Dist. vs Spec. Residual / Coffee [\%] / Cross Validation

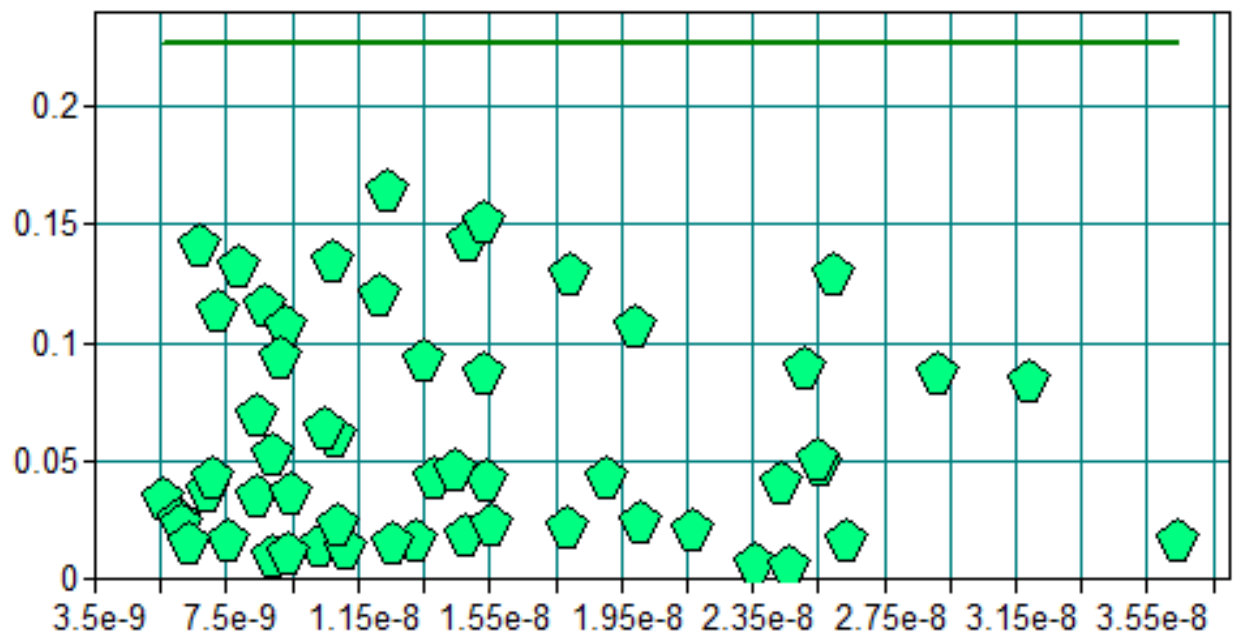

Rank: 3

Validation No $5+$ IncoffchnofdervMSC.q2

Score 2 vs Score $1 /$ Coffee [\%] / Cross Validation

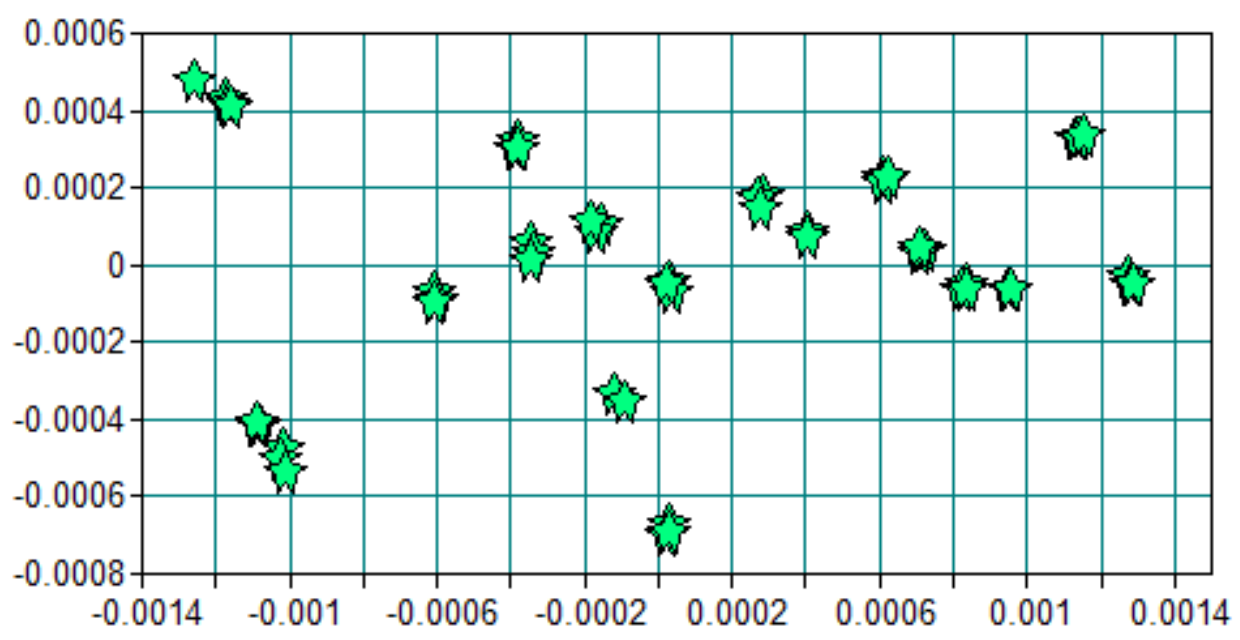

Validation No $5+$ IncoffchnofdervMSC.q2 
NIR spectra of instant coffee and chicory mixture with first derivative and vector normalization processing

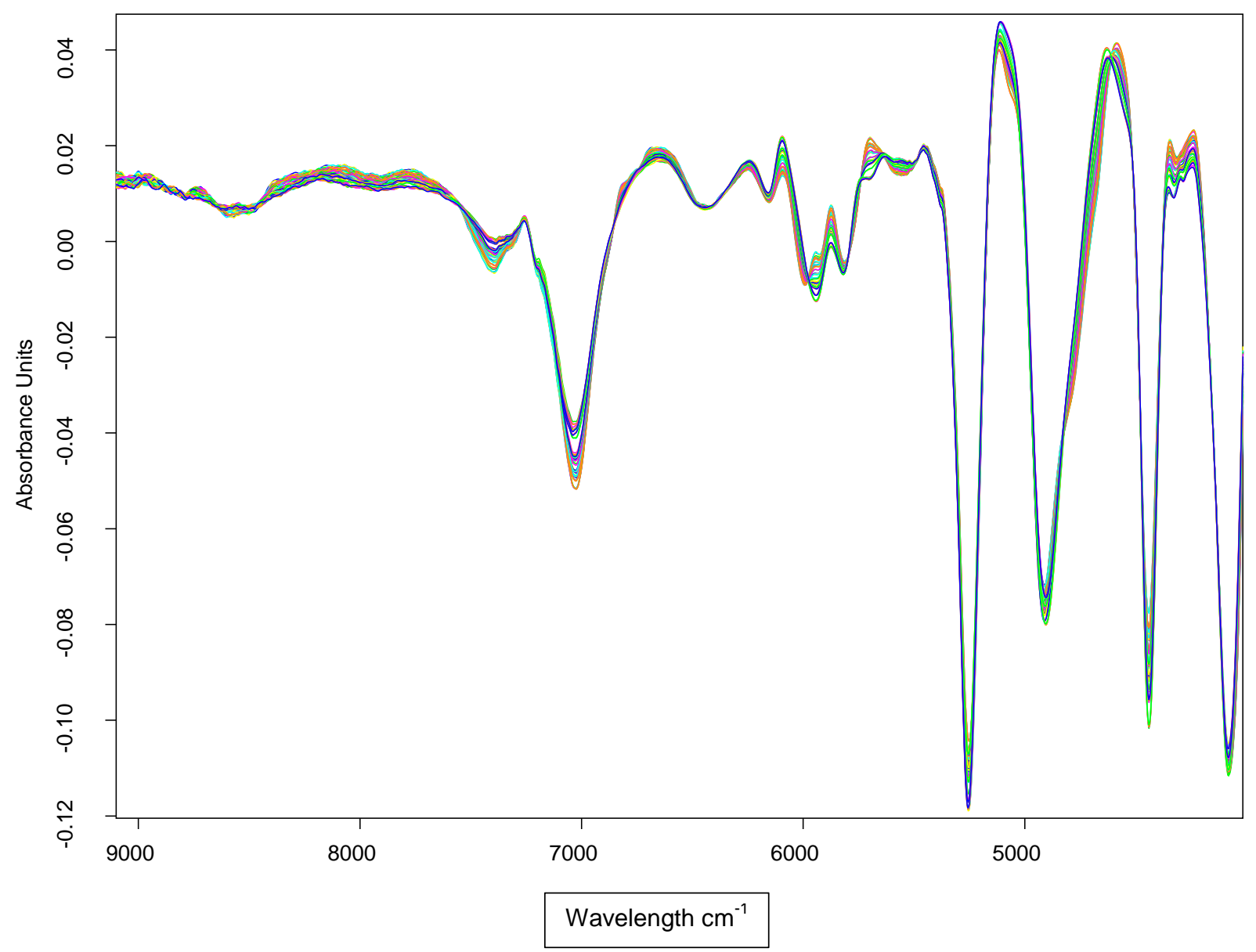


Prediction vs True / Coffee [\%] / Cross Validation

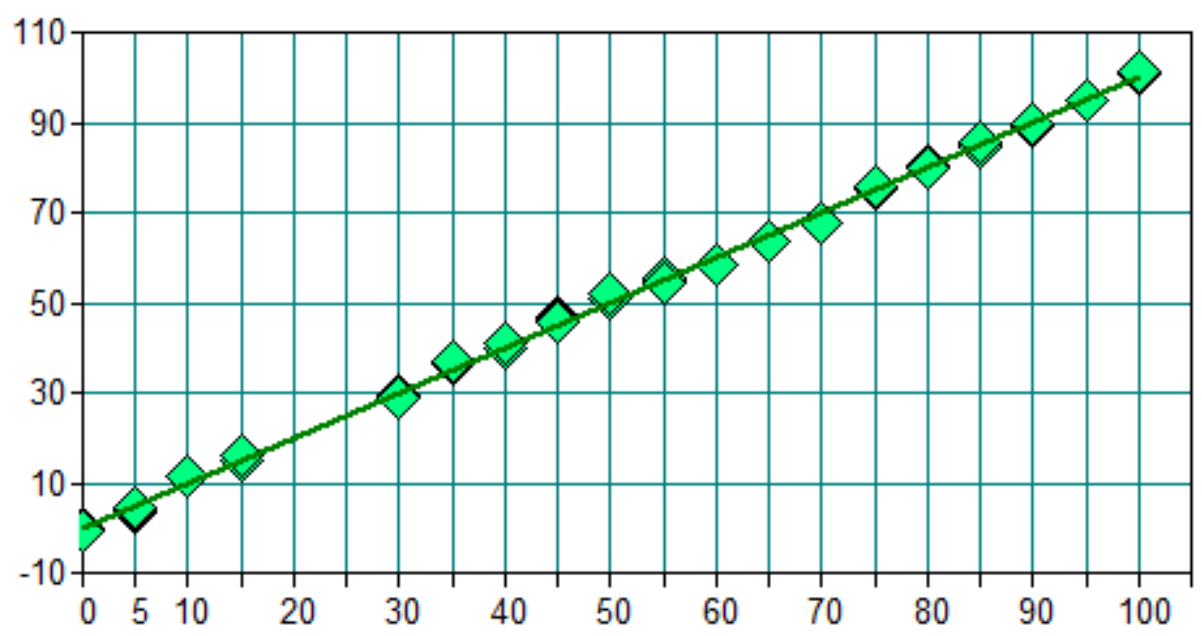

$\begin{array}{llll}\text { Rank: } 3 & R^{2}=99.88 & \text { RMSECV }=1.06 & \text { Bias: }-0.00138 \quad \text { RPD: } 29\end{array}$ Validation No $7+$ IncoffchnofdervSNV.q2

Difference vs True / Coffee [\%] / Cross Validation

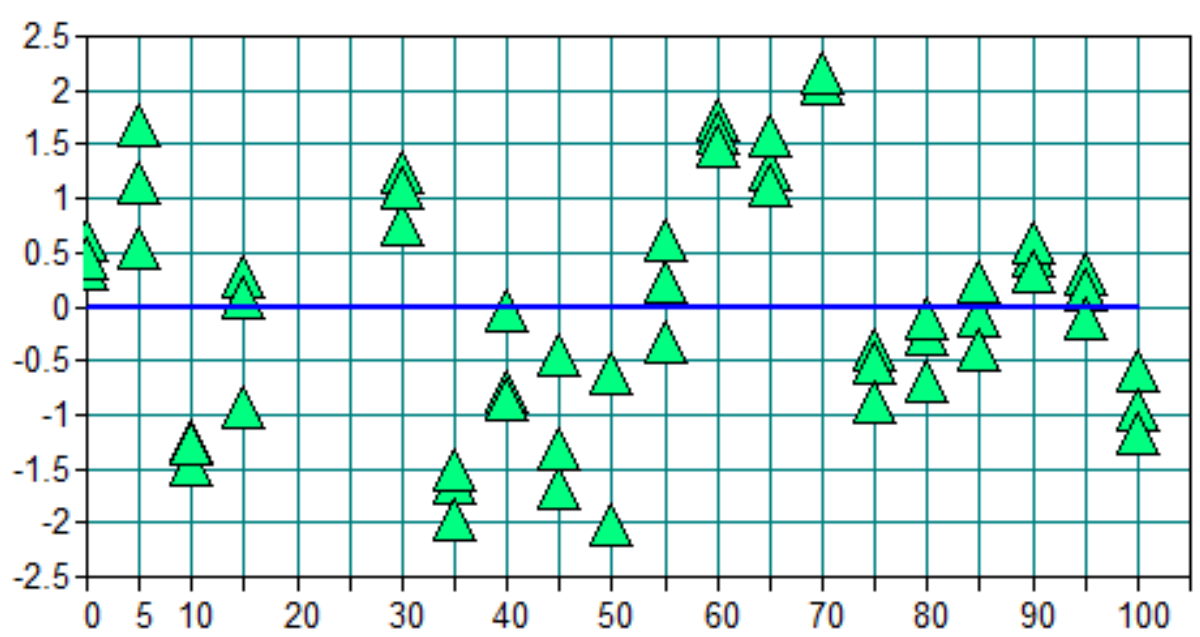

$\begin{array}{lllll}\text { Rank: } 3 & R^{2}=99.88 & \text { RMSECV }=1.06 & \text { Bias: }-0.00138 & \text { RPD: } 29\end{array}$

Validation No $7+$ IncoffchnofdervSNV.q2 
Mah. Dist. vs Spec. Residual / Coffee [\%] / Cross Validation

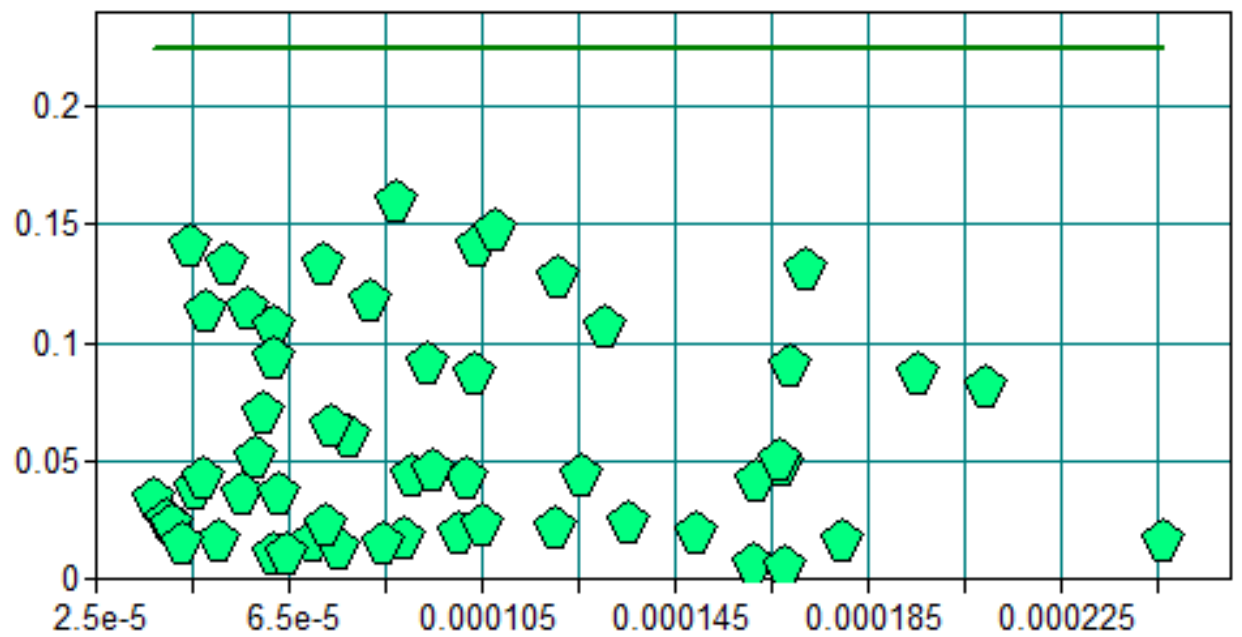

Rank: 3

Validation No $7+\quad$ IncoffchnofdervSNV.q2

Score 2 vs Score 1 / Coffee [\%] / Cross Validation

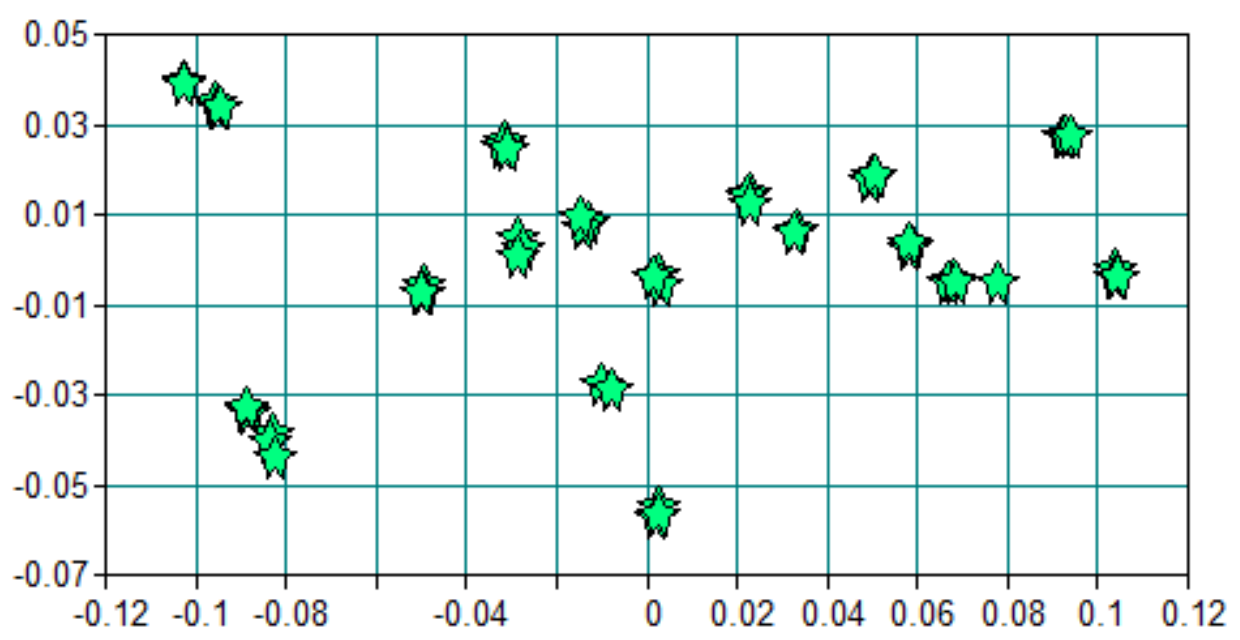

Validation No $7+\quad$ IncoffchnofdervSNV.q2 
NIR spectra of (Arabica plus Robusta) coffee and chicory mixture with no processing

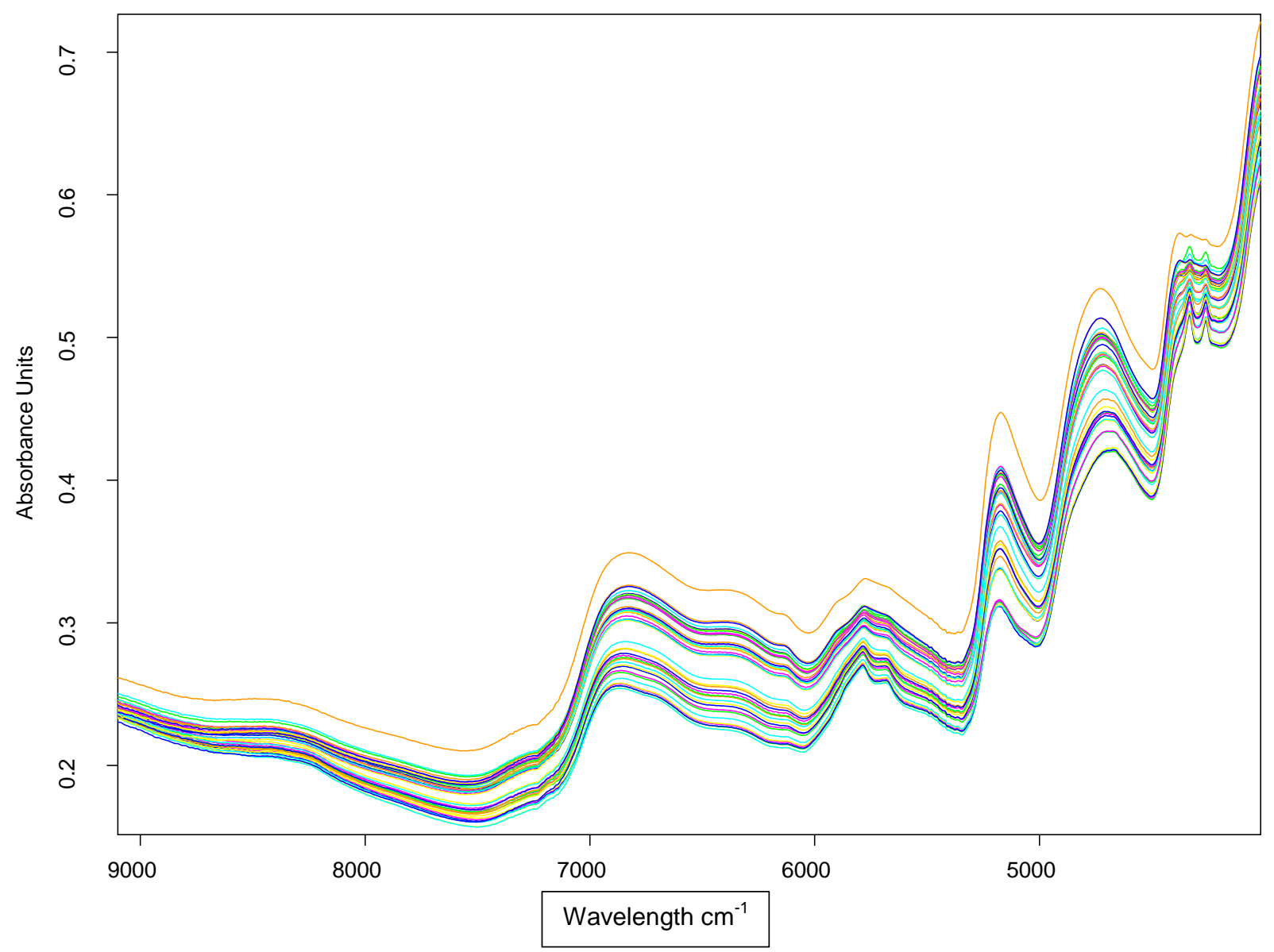


NIR spectra of (Arabica plus Robusta) coffee and chicory mixture with second derivative processing

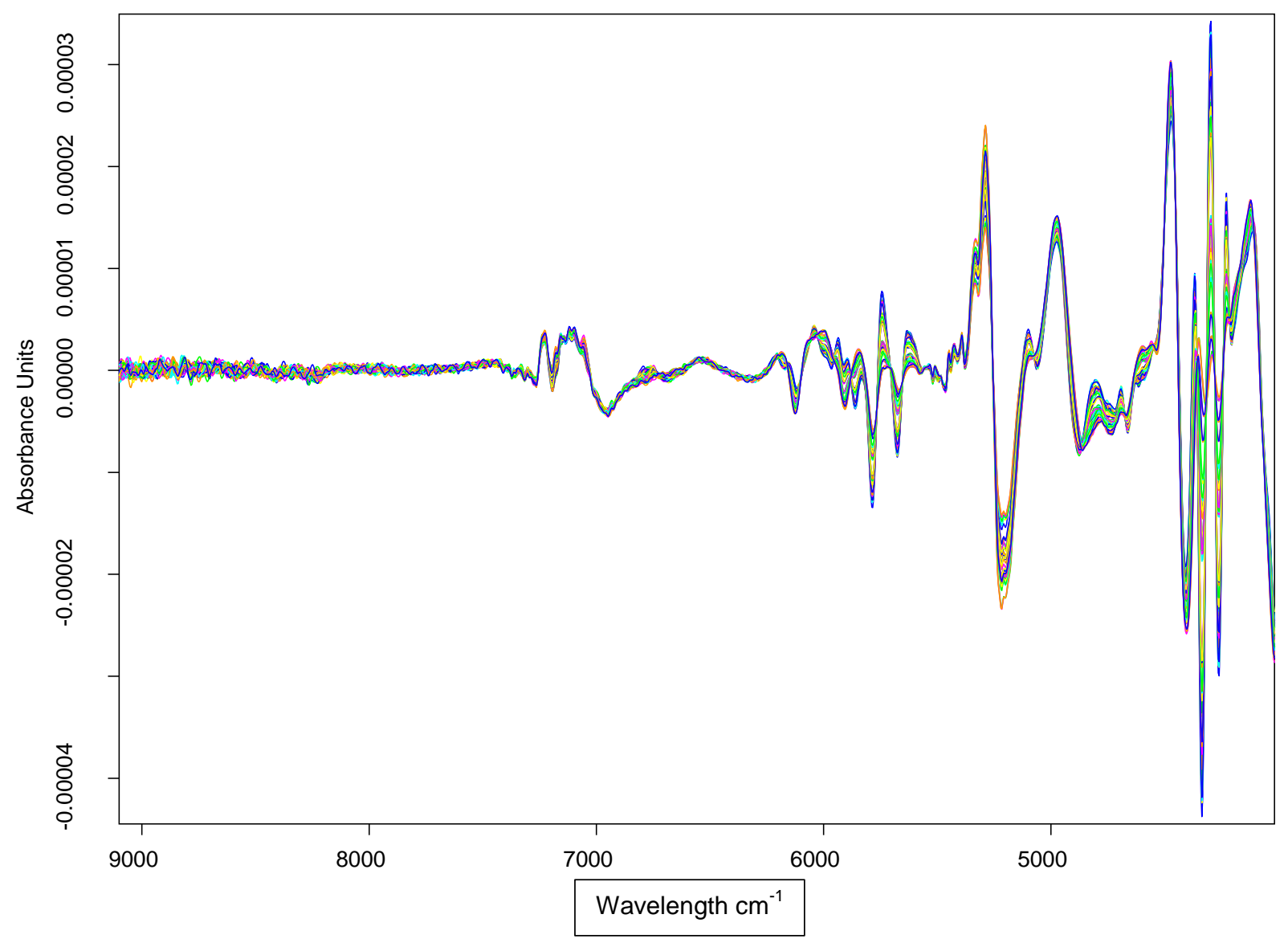


Prediction vs True / Coffee [\%] / Cross Validation

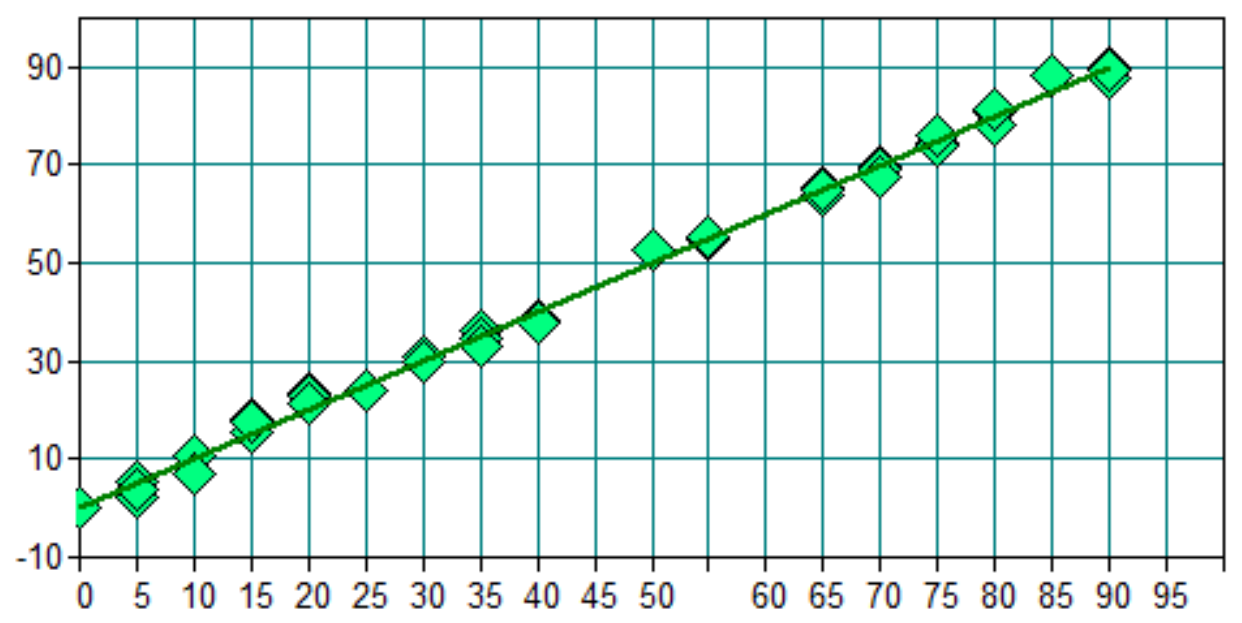

$\begin{array}{lllll}\text { Rank: } 4 & \mathrm{R}^{2}=99.68 & \text { RMSECV }=1.62 & \text { Bias: }-0.0107 & \text { RPD: } 17.8\end{array}$ Validation No $8+$ ArRobchSderv.q2

Difference vs True / Coffee [\%] / Cross Validation

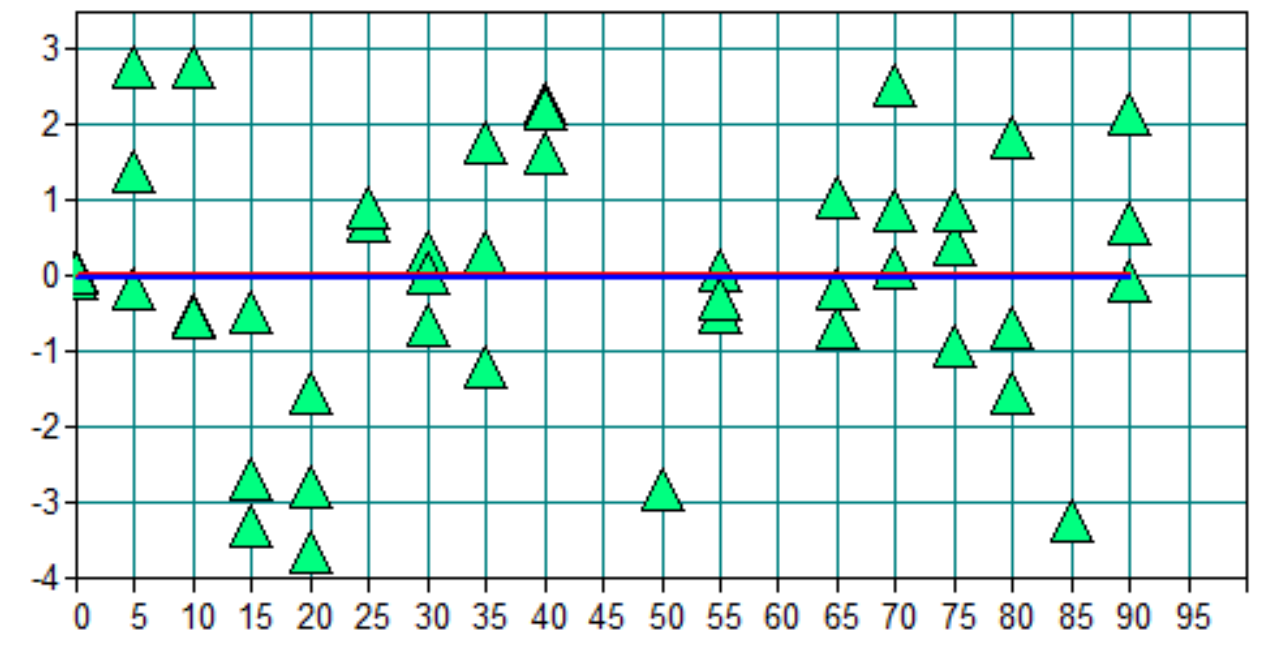

$\begin{array}{lllll}\text { Rank: } 4 & R^{2}=99.68 & \text { RMSECV }=1.62 & \text { Bias: }-0.0107 & \text { RPD: } 17.8\end{array}$

Validation No $8+$ ArRobchSderv.q2 
Mah. Dist. vs Spec. Residual / Coffee [\%] / Cross Validation

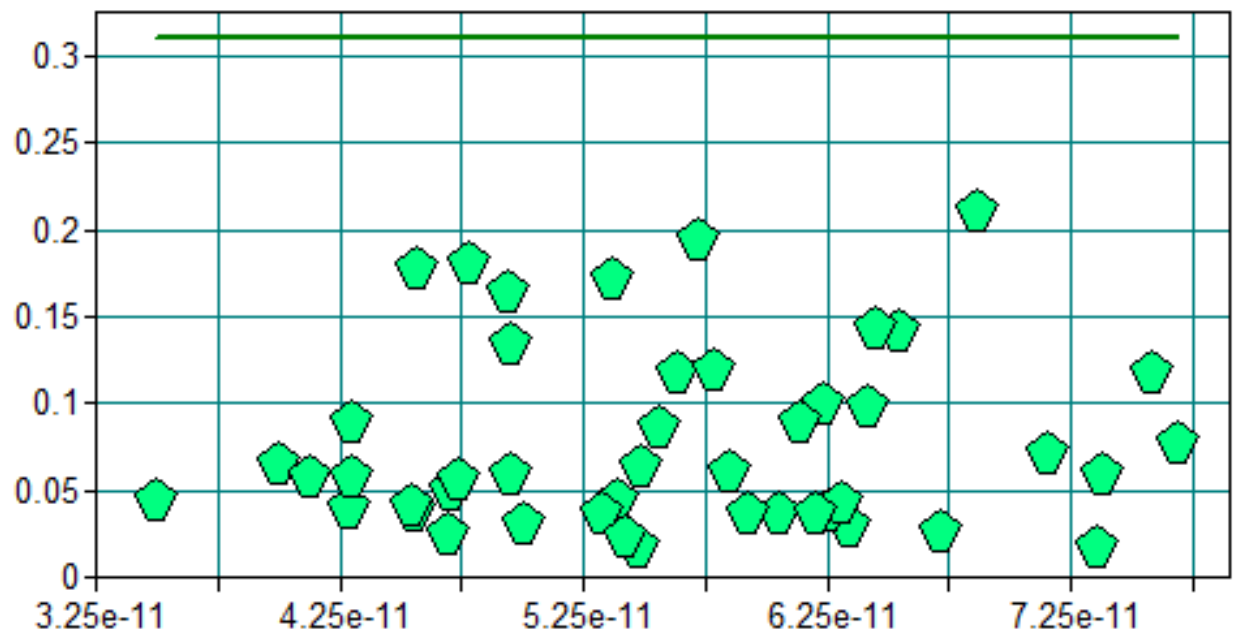

Rank: 4

Validation No $8+$ ArRobchSderv.q2

Score 2 vs Score $1 /$ Coffee [\%] / Cross Validation

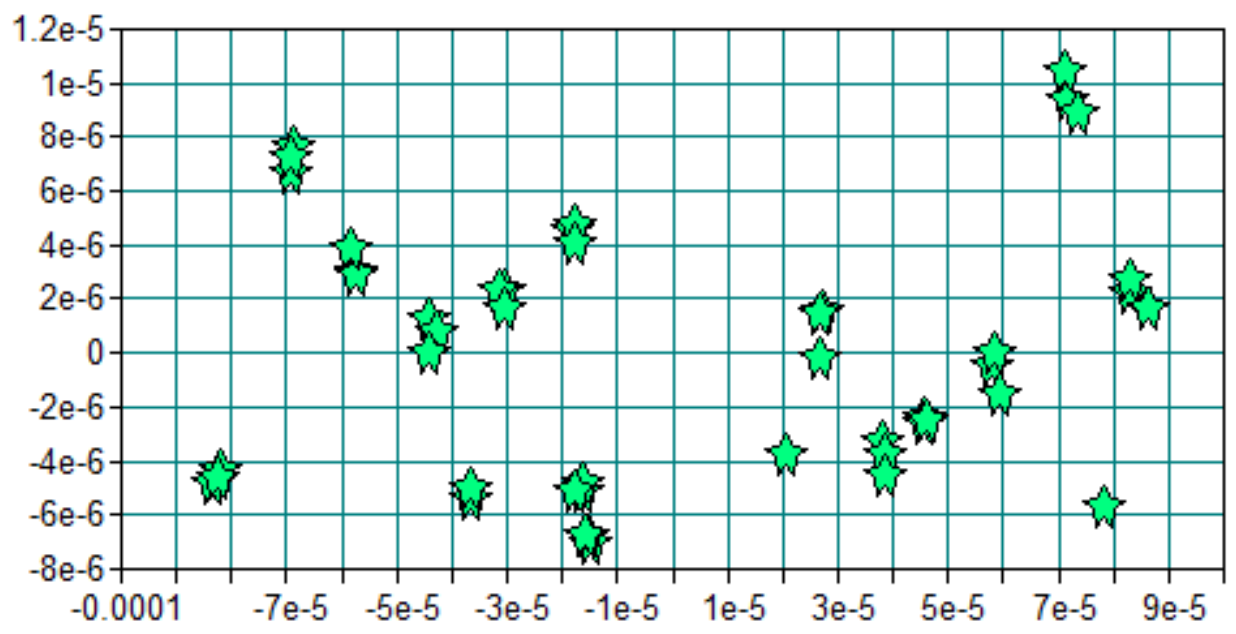

Validation No $8+$ ArRobchSderv.q2 
NIR spectra of Robusta coffee and chicory mixture with no processing

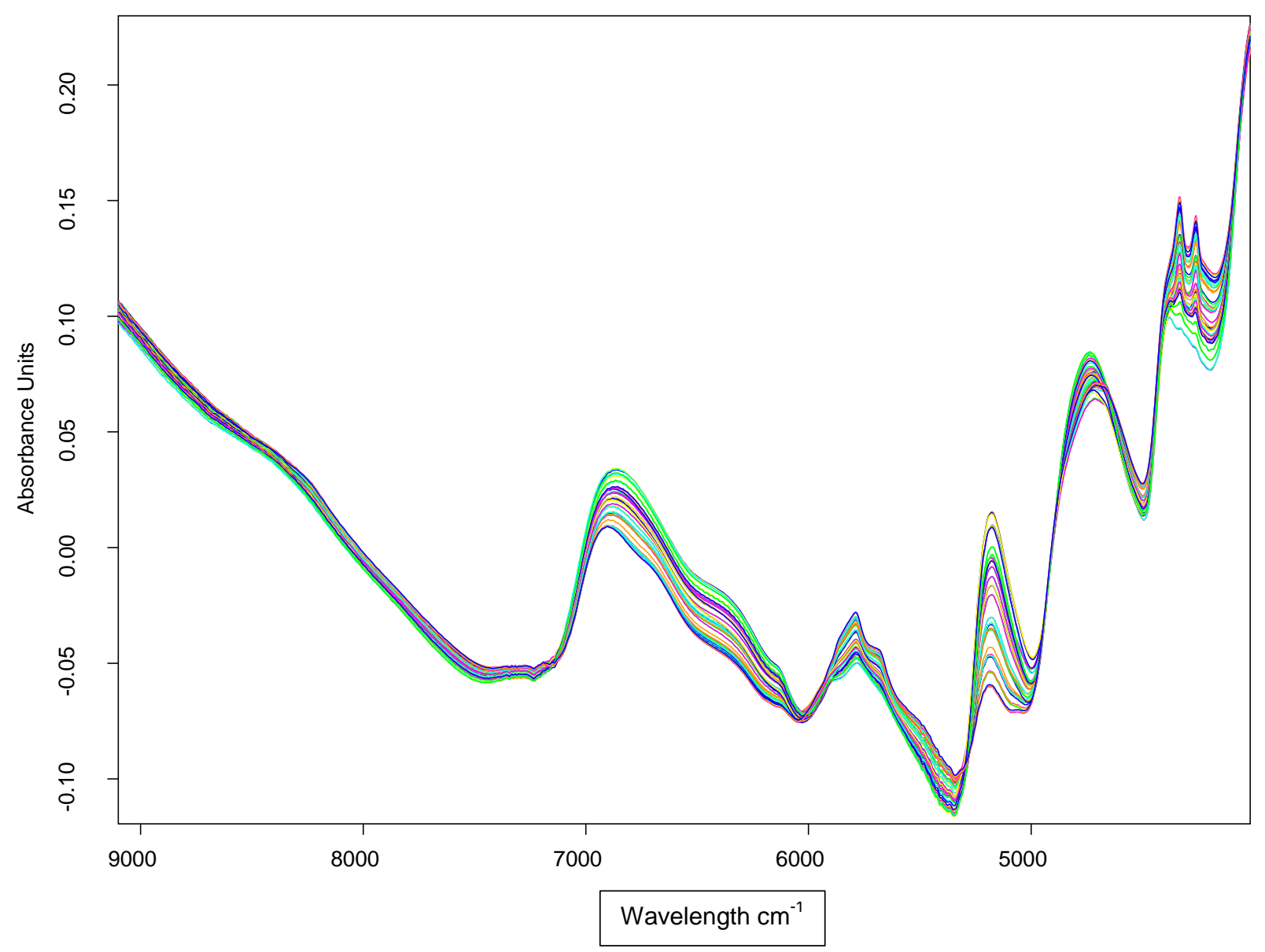


NIR spectra of Robusta coffee and chicory mixture with second derivative processing

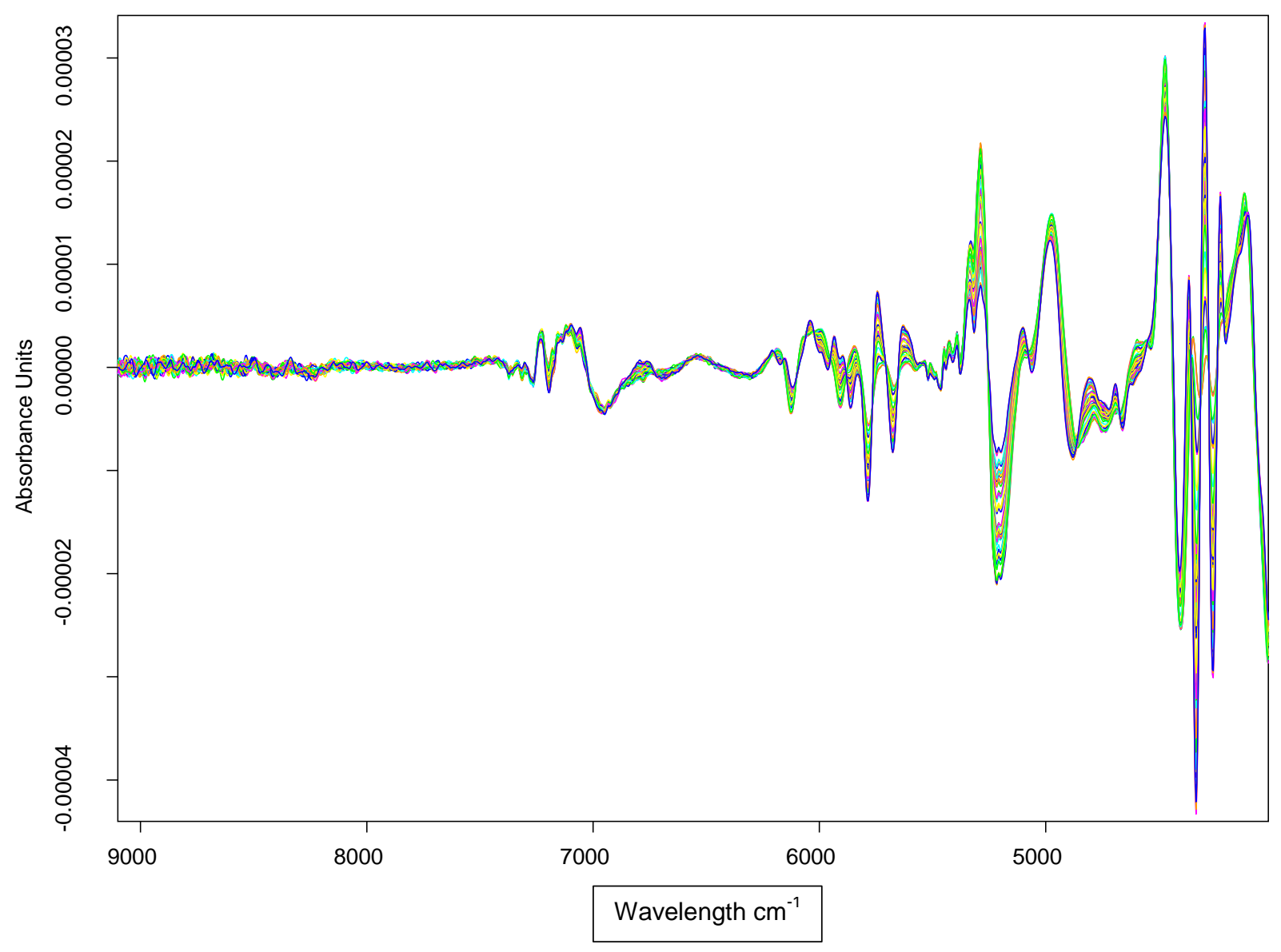


Prediction vs True / Coffee [\%] / Cross Validation

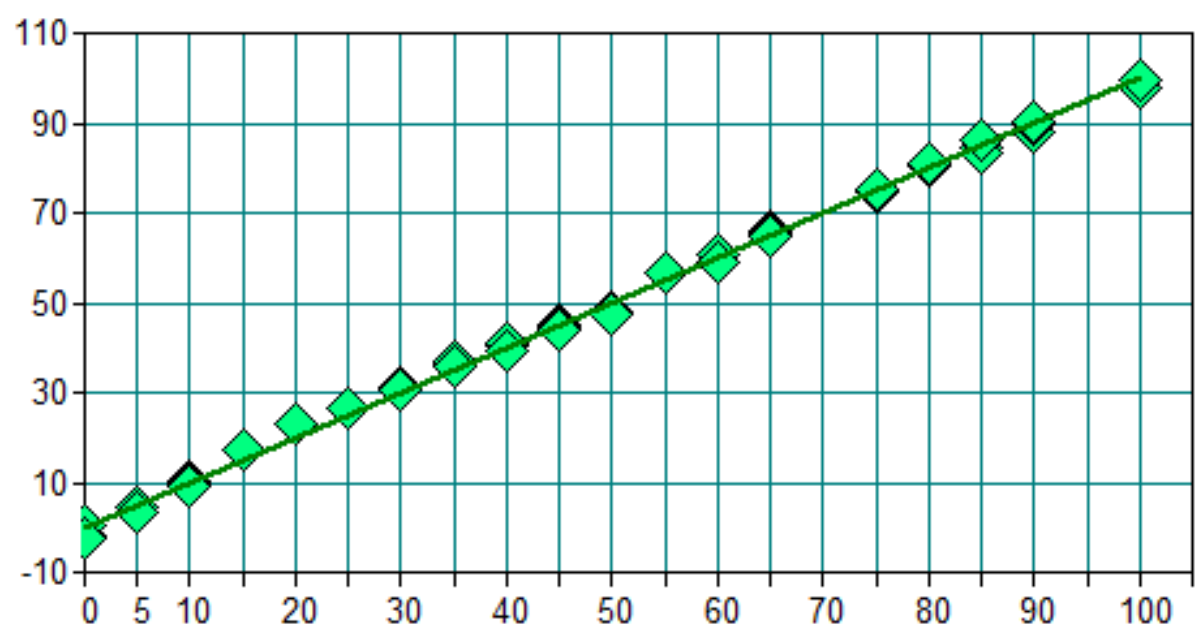

Rank: $5 \quad R^{2}=99.82 \quad$ RMSECV $=1.29 \quad$ Bias: $0.042 \quad$ RPD: 23.6 Validation No $11+$ Robchsder.q2

Difference vs True / Coffee [\%] / Cross Validation

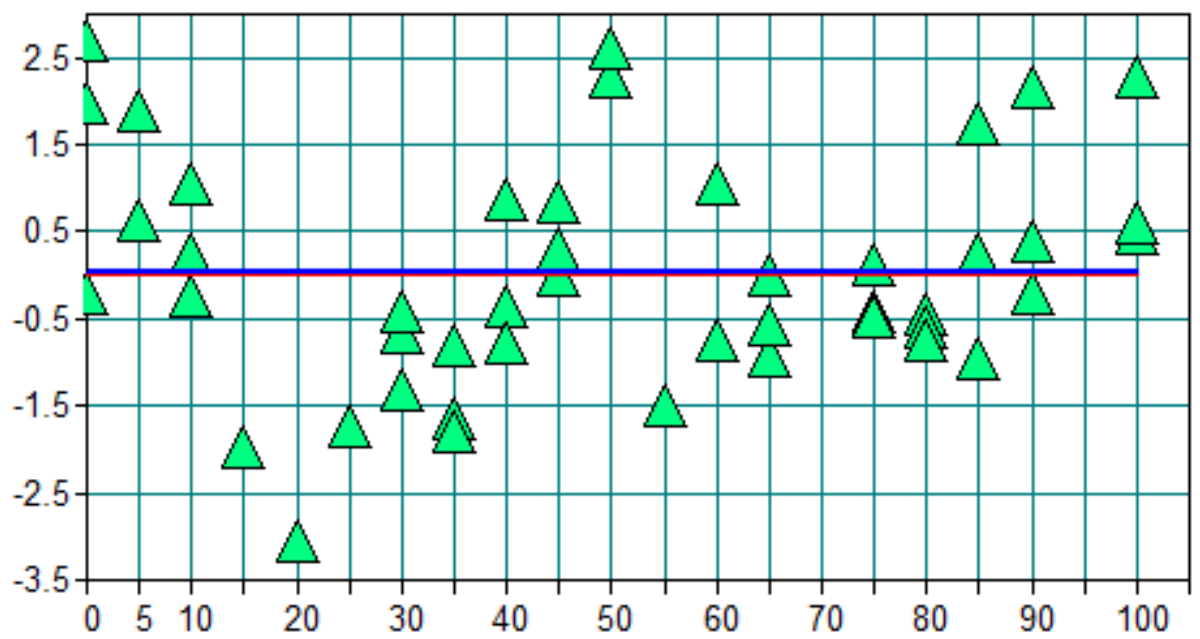

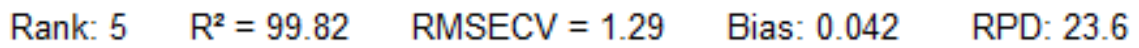

Validation No $11+$ Robchsder.q2 
Mah. Dist. vs Spec. Residual / Coffee [\%] / Cross Validation

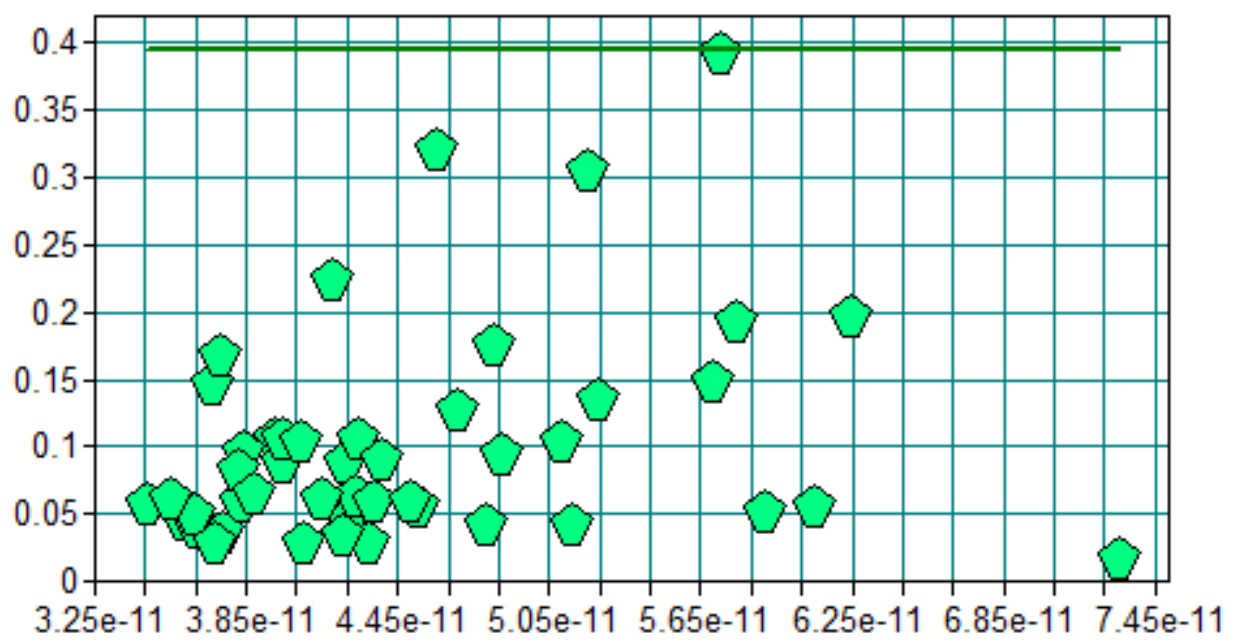

Rank: 5

Validation No $11+$ Robchsder.q2

Score 2 vs Score 1 / Coffee [\%] / Cross Validation

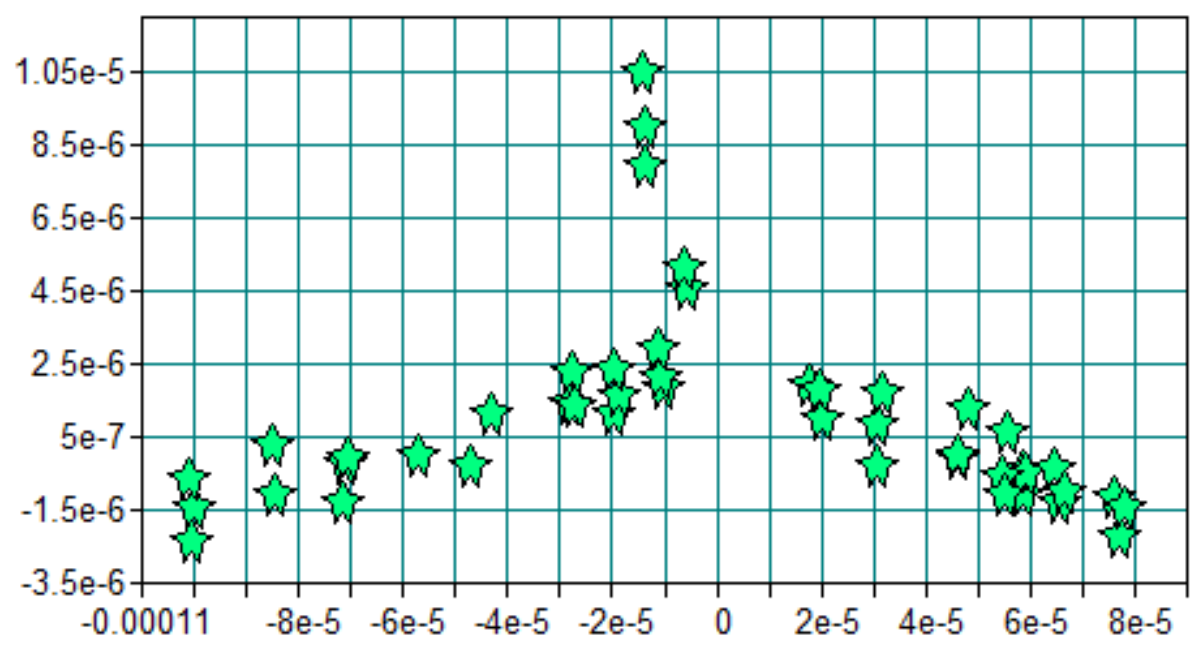

Validation No $11+$ Robchsder.q2 
NIR spectra of Arabica coffee and chicory mixture with no processing

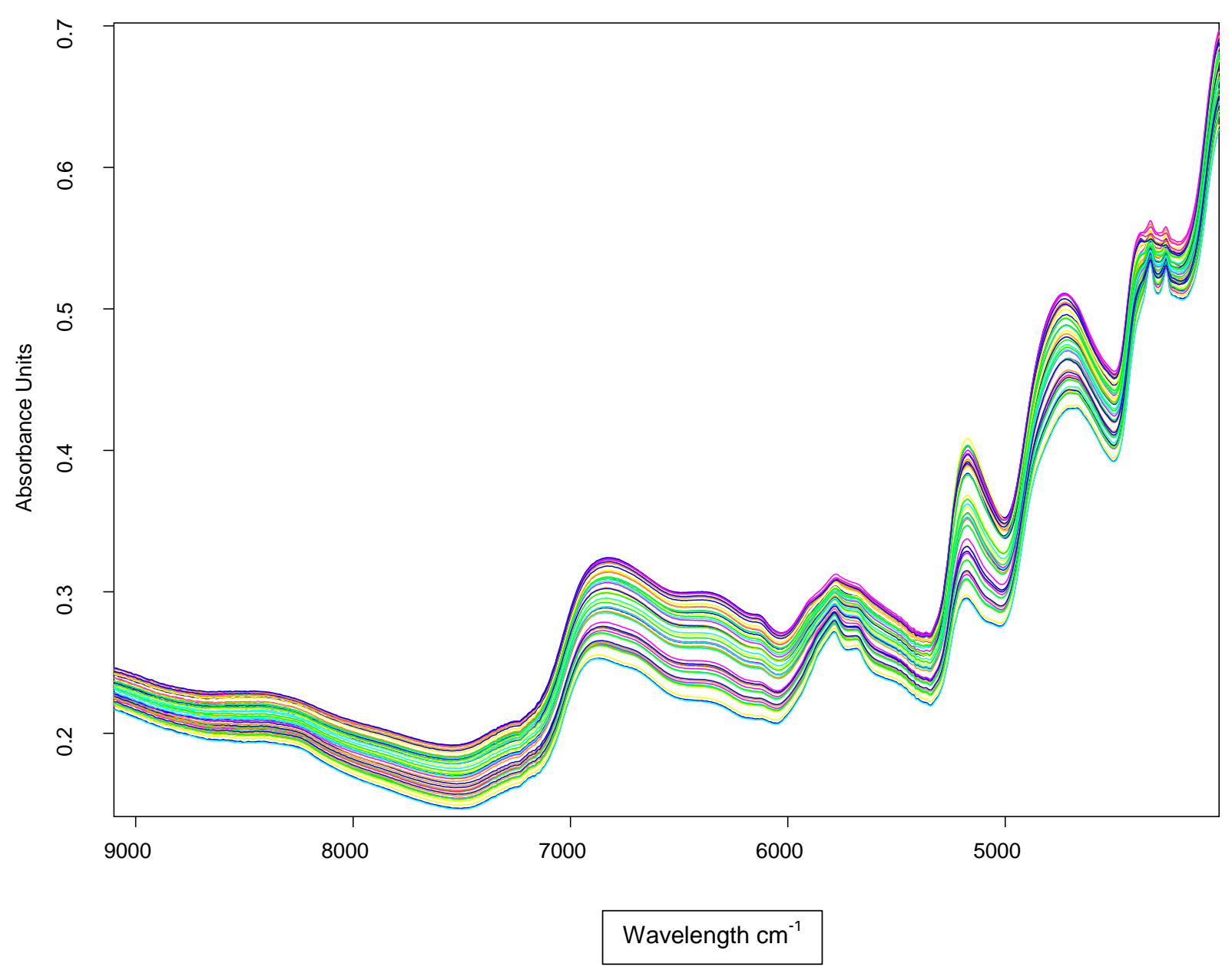

1 
NIR spectra of Arabica coffee and chicory mixture with second derivative processing

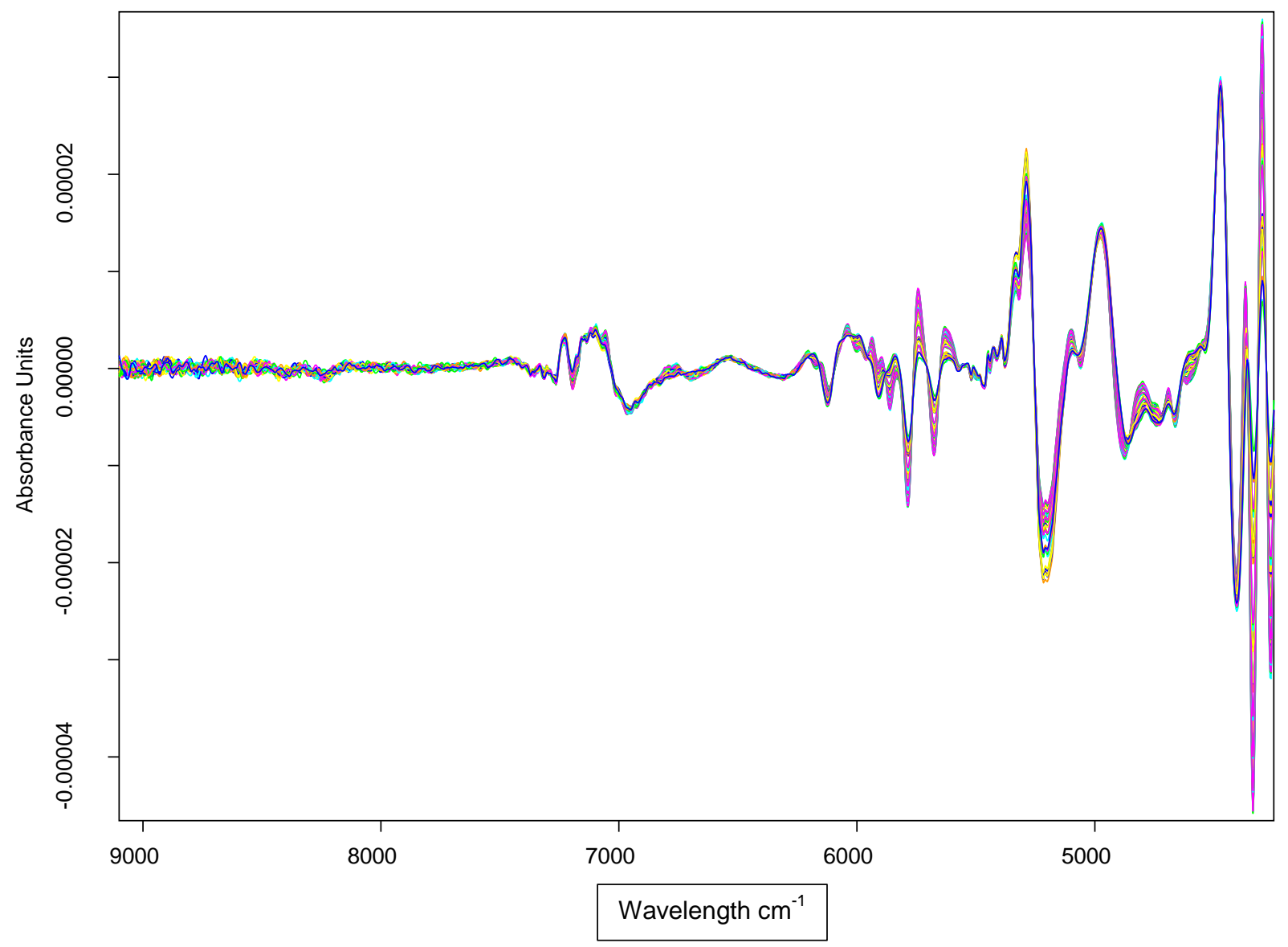


Prediction vs True / Coffee [\%] / Cross Validation

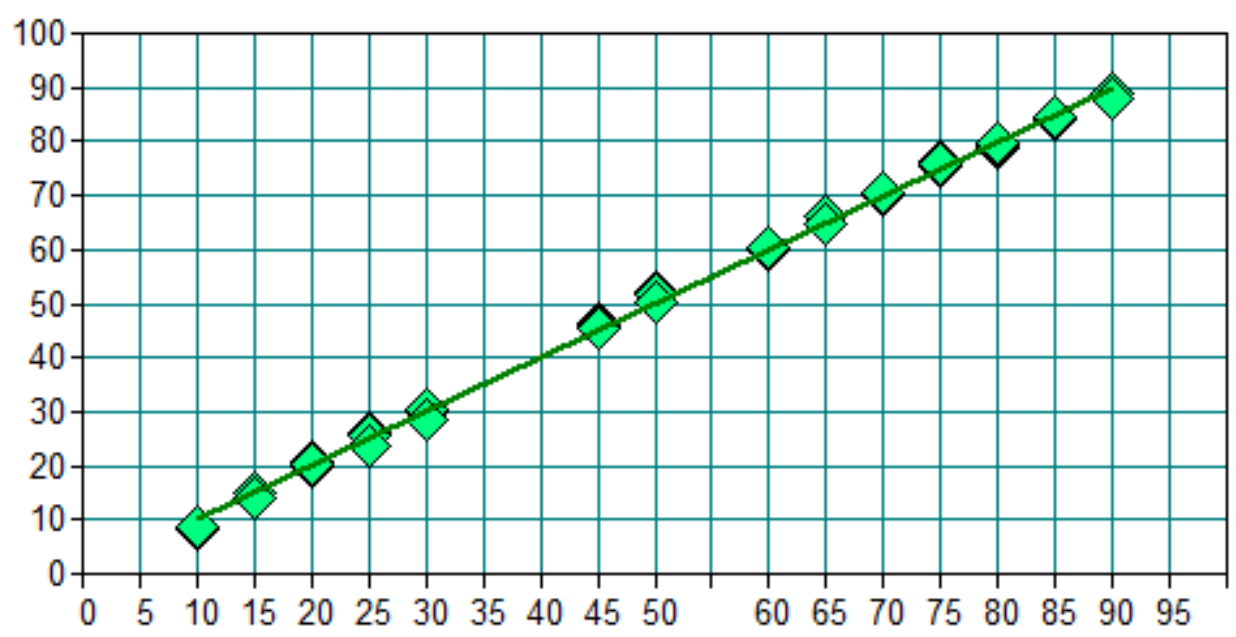

$\begin{array}{lllll}\text { Rank: } 3 & R^{2}=99.86 & \text { RMSECV }=0.969 & \text { Bias: } 0.0166 & \text { RPD: } 26.8\end{array}$

Validation No $13+$ Arbchsderrecent.q2

Difference vs True / Coffee [\%] / Cross Validation

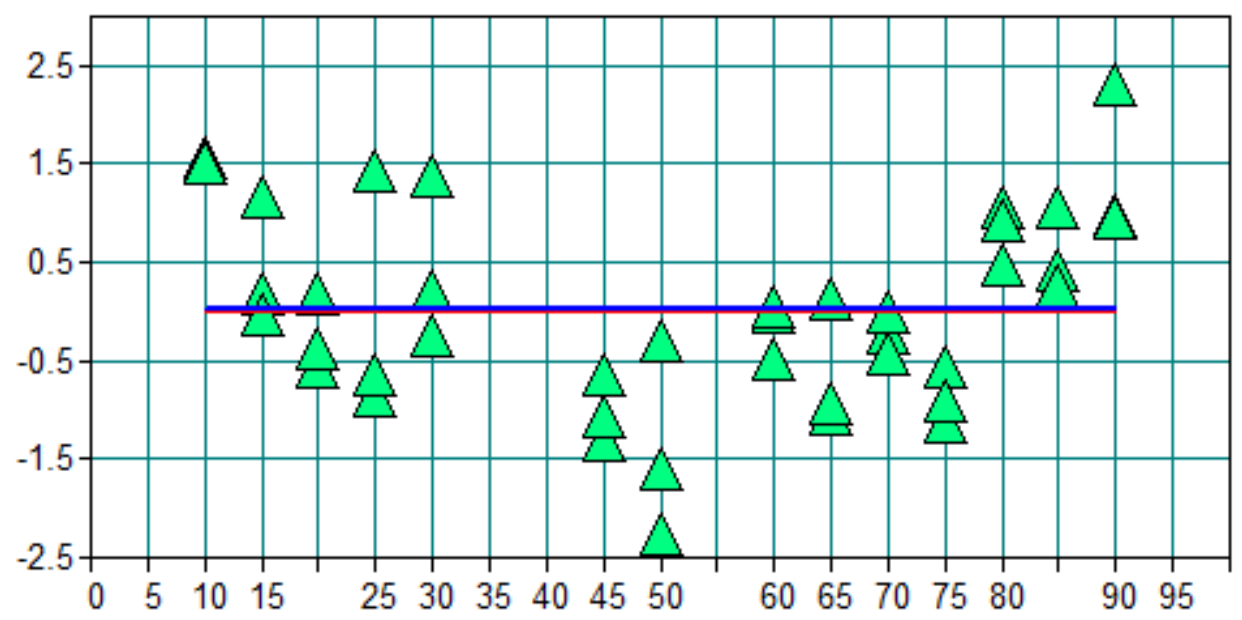

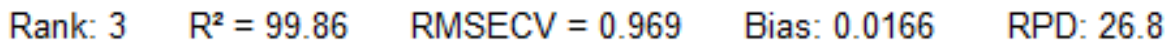

Validation No $13+$ Arbchsderrecent.q2 
Mah. Dist. vs Spec. Residual / Coffee [\%] / Cross Validation

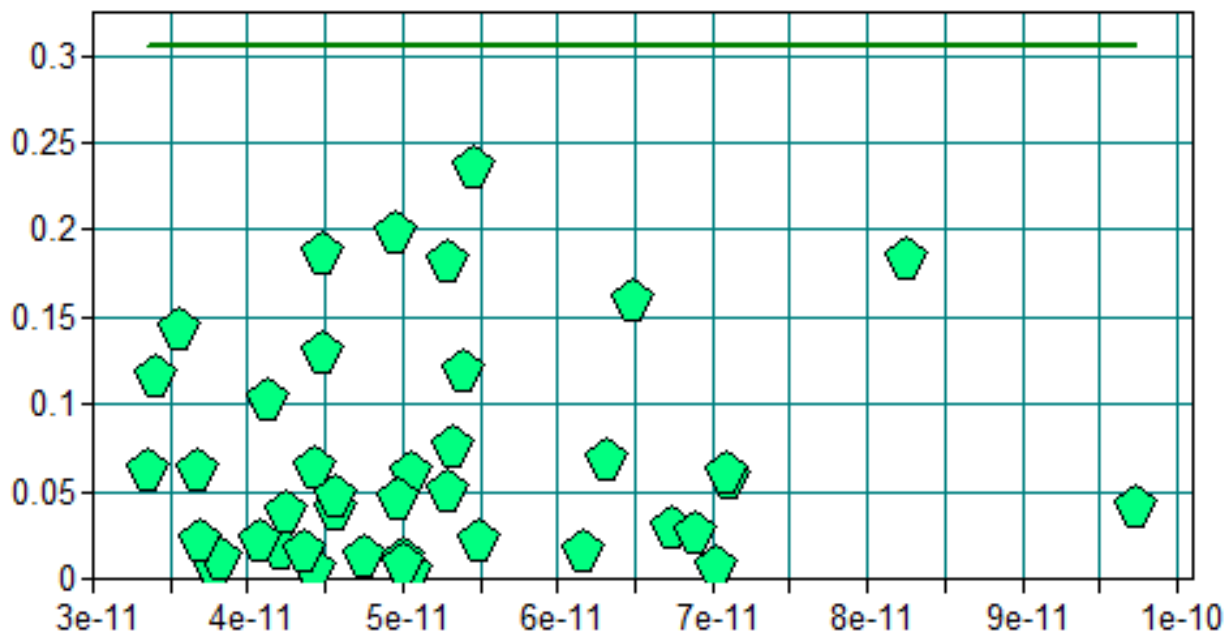

Rank: 3

Validation No $13+$ Arbchsderrecent.q2

Score 2 vs Score 1 / Coffee [\%] / Cross Validation

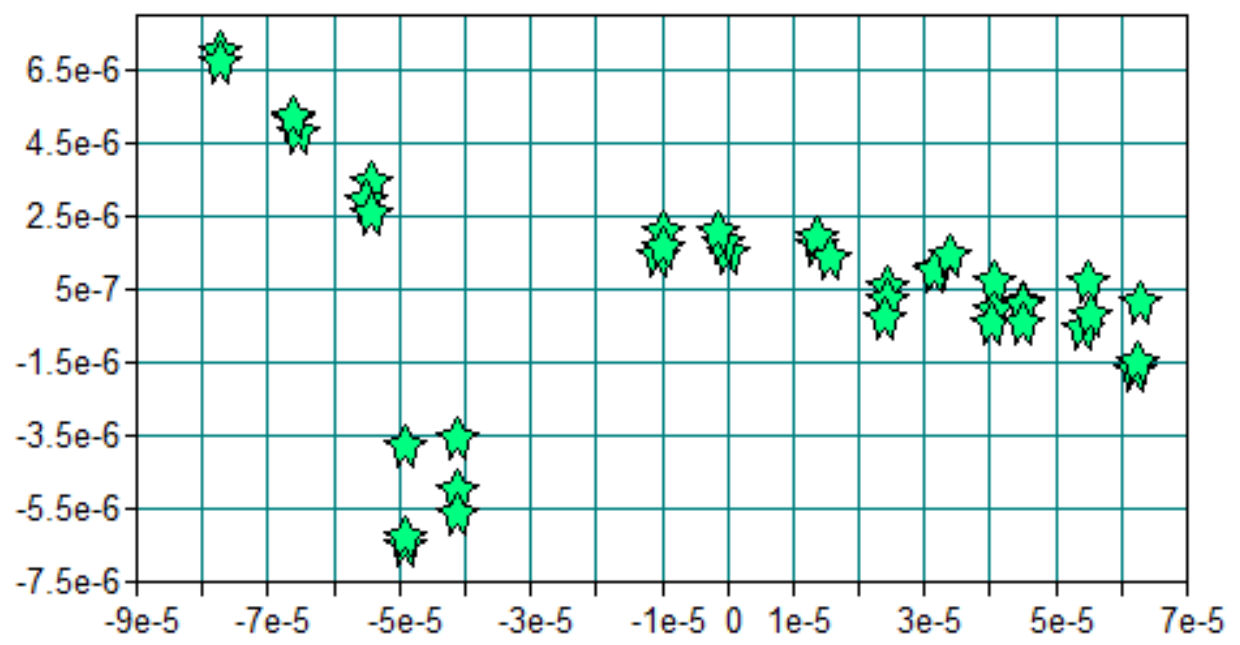

Validation No $13+$ Arbchsderrecent.q2 